\title{
A Two-layer Optimization Management Method for the Microgrid with Electric Vehicles
}

\author{
Zedong Zheng \\ Centre for Computational Intelligence (CCI) \\ School of Computer Science and Informatics \\ De Montfort University \\ The Gateway, Leicester LE1 9BH, UK \\ Email: donniezzd@gmail.com
}

\author{
Shengxiang Yang \\ Centre for Computational Intelligence (CCI) \\ School of Computer Science and Informatics \\ De Montfort University \\ The Gateway, Leicester LE1 9BH, UK \\ Email: syang@dmu.ac.uk
}

\begin{abstract}
The energy management of the microgrid (MG) with electric vehicles (EVs) is a large-scale optimization problem where the goal should take into account the performance and economic benefits of the power system while meeting the travel needs of EVs. Due to the development of vehicle to grid (V2G) technologies and demand response (DR), the relationship between EVs and MG becomes currently closer, which leads to a more complex situation. Therefore, the relationship of interest between MG and EVs has to be clarified to improve the performance of MG and EVs to achieve a win-win situation. This paper proposes a two-tier energy management strategy that considers the benefits for both MG and EVs. The first layer ensures the performance of the MG, while the second layer reduces the charging cost from the perspective of the car owners. In addition, based on the existence of uncertain parameters, mixed type variables and nonlinear constraints in the optimization problem, the differential evolution, stochastic search and greedy algorithm are used to analyze and find the optimal solution. Simulation results verify the effectiveness of the proposed strategy and solutions, which benefit both the MG and EV owners.
\end{abstract}

Index Terms-Optimization management, Microgrid, Electrical vehicle, Two-layer optimization, Differential evolution

\section{INTRODUCTION}

Energy shortages, environmental degradation and the economic downturn have created enormous challenges which have led to changes in energy industries [1]. The comprehensive smart grid has started replacing the traditional power grid with a single structure.

As basic units of the future integrated energy grid, microgrid (MG), usually contains distributed energy, energy storage devices, associated loads, energy converters and small distribution systems for monitoring and protecting equipment. A MG as an autonomous system that can achieve self-control, protection and management, can be operated in connecting with the main grid or in isolation. It is usually deployed around the users with low cost and small pollution, and is an effective way to achieve active power distribution network with flexible and efficient energy applications. Furthermore, the development and research of energy management strategies of MGs can fully promote the large-scale intervention of distributed energy, especially the environmental protection renewable energy source (RES), and find the highly reliable supply of various energy forms of the load.
Also, with the decline of battery cost and the development of rapid charging technology, electric vehicles (EVs) have taken up more and more market shares from traditional energy vehicles. The widespread use of EVs not only meets the commuting demand for social transportation but also reduces the greenhouse gas emissions generated by the transportation system. As a result, governments around the world encourage people using electric cars [2].

In addition, the emergence of demand response (DR) and vehicle to grid (V2G) technologies enables the bi-directional on demand flow of energy between EVs and the power grid. Furthermore, some of the new features including advanced smart metering, demand-side management and communication infrastructure are also added, giving many possibilities for integration the power grid with EVs. In this case, from the perspective of the power grid, EVs can provide auxiliary services for the power grid, which can be used as a dis-patchable flexible load or a dynamic energy storage device to improve the operation efficiency and stability of the power grid. Owners can change charging and discharging behaviours according to real-time information, meet their travel needs and also reduce their energy costs. Therefore, the combination of EVs and power grid is expected to improve their performance. However, the disordered EVs penetrate deeply into the power system, resulting in violent fluctuations of the power grid and affecting the security and stability of the power grid. Considering the complexity of the problem between the combination of the power system and EVs, an appropriate and effective intelligent energy scheduling management method is essential.

In recent years, many effective and feasible management methods have been reported. Li et al. [3] proposed a V2G operation time trigger scheduling management scheme based on the scenario that stochastic EVs are connected to a smart grid. EVs are used as a distributed energy storage system to achieve the balance between supplying and demanding of power grid. Morais et al. [4] proposed an effective optimization management method to optimize the demand curve of a smart grid by coordinating the charge and discharge of EVs to reduce the difference between the minimum and maximum demands.

Previous studies showed other benefits of integrating EVs into power systems, such as, assisting power grids to pro- 
mote economic and environmental benefits [5], [6], improving system efficiency, stability security [7]-[9] and the utilization rate of RESs [10], [11], and also increasing the income of EV owners and car parks [12], [13].

However, integrating massive EVs in a large power system is extremely challenging in terms of both model complexity and computational difficulty. In addition, how to use an appropriate charging and discharging strategy to schedule single EVs which can satisfy driving needs and owner benefits is also a complex question. Therefore, more and more researches begin to interpret the process of integrating $\mathrm{EVs}$ into the energy system from the perspective of MGs.

Kamankesh et al. [14] studied the optimal energy management of MGs on the basis of considering the uncertainty of EVs model. Three different charging modes were compared to understand the impact of different modes in the MG environment and developed a new robust and powerful symbiotic organisms search algorithm. Similarly, in view of the impact of EV charging on the optimal operation management of MG, Kavousi-Fard et al. [15] proposed a stochastic expert framework and an intelligent charging method that takes into account the energy requirement of EVs. In order to avoid the difference between the high and low peaks of power exchange between multiple MGs and main grid, Wang et al. [16] used the EVs as mobile battery devices to coordinate and dispatch with multiple MGs within a region. On this basis, a distributed scheduling strategy is proposed to reduce power cost.

It is clear from these studies which mainly focus on the optimal management strategy of MG energy. EVs are regarded as an adjustable load, and the research focuses on how to make EVs charge orderly. Some studies highlighted the importance in the coordination between the MG and EVs. In this relationship, research focuses on how to maximize the performance of EVs as mobile storage. It can also be found that most of the research is only conducted with one aspect of the interests of MG, EV owners or EV aggregate.

In this paper, a novel two-layer optimal management model is proposed to optimize the economic benefits of the MG and EVs. The upper layer regards EVs as mobile storage batteries with a variable capacity to minimize the daily cost of the MG. In the lower layer, the cost of each owner can be reduced as far as possible under the condition of meeting the travel and charging needs of EVs through a reasonable energy scheduling strategy. Meanwhile, considering the computational complexity of the model, a differential evolution (DE) algorithm, combined with stochastic optimization and greedy strategies, is used to solve the problem. Furthermore, the comparison of different solutions for the proposed model is discussed, and the economic relationship between an EV owner and the MG comprehensive benefit is studied.

The rest of this paper is organized as follows. Section II presents the operation strategy and problem formulation. Section III presents the proposed optimization method for solving the formulated problem. Section IV provides and discusses the simulation data and results. Section V concludes this paper.

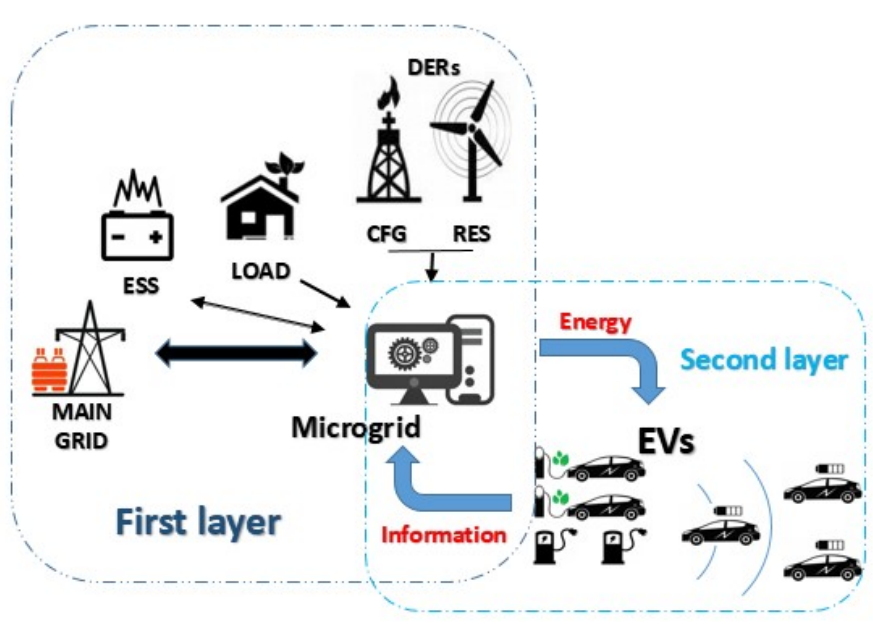

Fig. 1. Two-layer management methodology for the MG with EVs.

\section{METHODOLOGY AND FORMULATION}

The framework of the proposed two-layer optimization model is shown in Fig. 1, which includes four main parts: 1) DERs, including RESs and conventional fuel generator (CFG); 2) energy storage system (ESS); 3) EVs; 4) Load. The MG is connected to the main grid.

In order to save energy and protect the environment, the green RES is prioritized to be used to meet the load demand of the MG, while EV aggregators and ESS are used to balance the volatility from RES. When the output power of RES is insufficient, CFG and main grid act as additional energy supplements to the MG. In addition, under certain conditions, the surplus power of the MG can also be sold to the main grid or EVs.

In current work, all the connected EVs participating in the scheduling are assumed to provide corresponding information, such as the arrival time, departure time, and information and parameters of car batteries. In addition, in view of activating the enthusiasm of EV owners in participation and reducing their mileage anxiety, in this paper, the charging requirement and cost of each EV will be considered with the prediction price at first, and then considers the auxiliary services provided by EVs. This means that the car owner first decides the specific period for charging, which also means the car will no longer provide auxiliary services during that period.

In the first layer of the proposed model, all connected available EVs will be treated as mobile ESS units participating in the optimize operation of a MG. Therefore, the available capacity of the mobile ESS will be one of important constraints for the MG optimization model. In the second layer, the schedule of charging or discharging for each EV will be determined by real price, battery status and the results of the optimized operation of the first layer. The two layers are described in detail below. For the convenience of readers, Table I provides a list of notations with brief descriptions. 
TABLE I

NOTATION AND DESCRIPTION

\begin{tabular}{|l|l|}
\hline Notation & Description \\
\hline$C_{d a i l y}$ & The daily cost of MG \\
\hline$C_{e v}$ & The cost of each EV \\
\hline$P_{E G}^{t}$ & The electric price at period $t$ \\
\hline$P_{f u e l}^{i, t}$ & The fuel price of the $i$ th DER at the period $t$ \\
\hline$P_{D E R}^{i}$ & The start-up/ stop price of DER $i$ \\
\hline$P_{o m}^{i, t}$ & The maintain price of DER $i$ \\
\hline$P_{e m}^{i, t}$ & The pollution control price of DER $i$ \\
\hline$P_{E V a g g}^{t}$ & The price of auxiliary service from the EV aggregate at the period $t$ \\
\hline$P_{E V r e q}^{t}$ & The price of charging for EV aggregate at the period $t$ \\
\hline$P_{E S S o m}^{n, t}$ & The maintain price of the $n$th ESS \\
\hline$E_{E G}^{t}$ & The exchange power with the main grid \\
\hline$F_{f u e l}^{t}\left(E_{D E R}^{i, t}\right)$ & The amount of fuel consumed of DER i at the period $t$ \\
\hline$Q_{e m}^{i, t}\left(E_{D E R}^{i, t}\right)$ & The amount of pollution gas of DER $i$ at the period $t$ \\
\hline$E_{E V a g g}^{t}$ & The auxiliary service power from the EV aggregate \\
\hline$E_{E S S}^{n, t}$ & The exchanged power of ESS $n$ at the period $t$ \\
\hline$E_{E V r e q}^{t}$ & The total power requirement for the EV aggregate \\
\hline$E_{C F G}^{t}$ & The power from CFG generation at the period $t$ \\
\hline$S_{O N}^{i, t}, S_{O F F}^{i, t}$ & Binary variable: The statues of DER $i$ at the period $t$ \\
\hline$S O C_{C A P}^{k, m a x}, S O C_{C A P}^{k, m i n}$ & The maximum and minimum limit of the batteries $S O C$ for each car \\
\hline$S O C^{t, k}$ & The $S O C$ of the car $k$ at the period $t$ \\
\hline$S O C_{a r r}^{k}$ & The $S O C$ of the car $k$ when it arrives \\
\hline$S O C_{d e p}^{k}$ & The $S O C$ of the car $k$ when it departure \\
\hline$t_{a r r}^{k}$ & The arrival time of the car $k$ \\
\hline$t_{d e p}^{k}$ & The departure time of the car $k$ \\
\hline$t_{r e q}^{k}$ & The charging time of the car $k$ \\
\hline$E V_{C A P}^{k}$ & The rating power of car $k$ \\
\hline$E_{L O A D}^{t}$ & Microgrid load at the period $t$ \\
\hline$E_{E V \text { service }}^{t, k}$ & The power for the auxiliary service from car $k$ at the period $t$ \\
\hline$E_{E V r e q}^{t, k}$ & The power requirement for the car $k$ at the period $t$ \\
\hline$l^{t}$ & Binary variable: The statues of EV \\
\hline$t, T$ & Index for periods, Number of periods \\
\hline$k, K$ & Index for EVs, Number of EVs \\
\hline$i, I$ & Index for DER units, Number of DERs \\
\hline$n, N$ & Index for ESS units, Number of ESSs \\
\hline & \\
\hline & \\
\hline &
\end{tabular}

\section{A. First layer: $M G$ daily cost minimization}

The cost of MG is calculated by the operating cost and revenue of MG. The cost-function can be expressed as Eq. (1) and is comprised of four parts. They are the exchange cost with the main grid, the energy generation cost of DERs(including CFG and RES), the interaction cost of ESSs and EVs.

$$
\begin{aligned}
\min C_{\text {daily }}= & \sum_{t=1}^{T}\left\{P_{E G}^{t} \cdot E_{E G}^{t}\right. \\
& +\sum_{i=1}^{I}\left\{P_{\text {fuel }}^{i, t} \cdot F_{\text {fuel }}^{t}\left(E_{D E R}^{i, t}\right)\right. \\
& +P_{D E R}^{t} \cdot\left(s_{O N}^{i, t}+s_{O F F}^{i, t}\right)+P_{\text {om }}^{i} \cdot E_{D E R}^{i, t} \\
& \left.+P_{e m}^{i, t} \cdot Q_{e m}^{i, t}\left(E_{D E R}^{i, t}\right)\right\} \\
& +P_{E V a g g}^{t} \cdot\left|E_{E V a g g}^{t}\right| \\
& \left.+\sum_{n=1}^{N} P_{E S S o m}^{n, t} \cdot\left|E_{E S S}^{n, t}\right|\right\}
\end{aligned}
$$

Considering that the provision of auxiliary services for the MG by EVs will accelerate the aging of the battery, the MG should compensate the EVs which are involved in dispatching and add the compensation fees to the calculation of operating cost.

\section{Constraints:}

An important constraint to be satisfied in the MG operation problem is regarding the active power balance at any time. The active power balance constraint can be expressed as follows:

$$
\begin{aligned}
E_{E G}^{t}+\sum_{i=1}^{I}\left(E_{D E R}^{i, t}\right) & +E_{E V a g g}^{t}+\sum_{n=1}^{N}\left(E_{E S S}^{n, t}\right) \\
& =E_{\text {LOAD }}^{t}+E_{E V r e q}^{t} \quad \forall t \in T
\end{aligned}
$$

As previously described, on the first step, the available mobile ESS (EV aggregator) capacity is an important constraint, which can be expressed as follows: 


$$
\begin{aligned}
& \text { charging } \\
& -\sum_{\substack{t=1 \\
\text { discharging }}}^{K}\left(S O C^{t, k}-S O C_{C A P}^{k, \text { min }}\right) \cdot l^{t} \leq \frac{E_{E V a g g}^{t}}{E V_{C A P}^{k}}<0 \\
& 0<\frac{E_{E V a g g}^{t}}{E V_{C A P}^{k}} \leq \sum_{t=1}^{T} \sum_{k=1}^{K}\left(S O C_{C A P}^{k, \max }-S O C^{t, k}\right) \cdot l^{t}
\end{aligned}
$$

In Eq. (3), when EVs as mobile ESS for the MG (charging), the value is positive; while providing energy supplement service (discharging), the value is negative. When the value is zero, the MG does not use the auxiliary service of EVs.

In addition, EV aggregators involved in optimizing the management need to take into account the battery capacity of each EV. Constraints in Eq. (4) makes sure that EV battery SOC is maintained in a safe range. Constraints in Eq. (5) describes the initial state of the battery when the $\mathrm{EV}$ is connected. Considering the travel needs of the EV owner, constraints (6) indicates each EV battery SOC should not be less than the desired value at the departure time. And $l^{t}$ represents the status of EV, as defined in Eq. (7).

$$
\begin{gathered}
S O C_{C A P}^{k, \text { min }} \leq S O C^{t, k} \leq S O C_{C A P}^{k, \max } \\
S O C^{t, k}=S O C_{a r r}^{k} \quad t=t_{\text {arr }}^{k}, \forall k \in K \\
S O C^{t, k} \geq S O C_{d e p}^{k} \quad t=t_{d e p}^{k}, \forall k \in K \\
l^{t}=\left\{\begin{array}{ll}
0 & \text { if } t<t_{\text {arr }}^{k} \text { or } t_{\text {dep }}^{k}<t \text { or } t=t_{r e q}^{k} \\
1 \quad \text { if } t_{\text {arr }}^{k} \leq t \leq t_{\text {dep }}^{k}
\end{array} \quad \forall k \in K\right.
\end{gathered}
$$

\section{B. Second layer: each EV cost minimization}

The second layer of optimization is a resource allocation problem. The objective is to minimize EV owners' cost, which consists of two terms: the first term represents the compensation income for participating in the MG auxiliary services, and the second term refers to the energy charging fees which meet the power requirement of the owner. The objective function of each EV is formulated as in Eq. (8):

$$
\begin{aligned}
\min C_{e v} & =\sum_{t=1}^{T} \sum_{k=1}^{K}\left\{P_{E V \text { agg }}^{t} \cdot\left|E_{E V \text { service }}^{t, k}\right| \cdot l^{t} \cdot \Delta T\right. \\
& \left.-P_{E V r e q}^{t} \cdot E_{E V r e q}^{t, k}\right\}
\end{aligned}
$$

\section{Constraints:}

It is indicated in Eq. (9) that, the total energy of participating in MG auxiliary service is equal to $E_{E V a g g}^{t}$ which is of an optimal result from the upper layer. And each EV has the same constraints subject to Eqs. (4)-(6).

$$
\sum_{k=1}^{K} E_{E V \text { service }}^{t, k}=E_{E V a g g}^{t}
$$

The relationship between energy allocation and EV battery $S O C$ at each connection time interval is defined in Eq. (10).

$$
\begin{aligned}
S O C^{t+1, k}=S O C^{t, k}+\frac{E_{E V \text { service }}^{t, k} \cdot l^{t}+E_{E V r e q}^{t, k}}{E V_{C A P}^{k}} \\
\forall k \in K \quad \forall T \quad \forall t \in T \quad \text { (10) }
\end{aligned}
$$

\section{Proposed Optimization Method}

It can be seen from the description and models in Section II, the optimization problem of the first layer is a non-convex non-linear one. Therefore, traditional optimization methods cannot deal with it [17]. Evolutionary algorithms (EAs) have the potential to solve this optimization problem since they are a kind of population-based heuristic search methods which only require the objective function values and do not need the gradient information. In addition, the problem is an optimization problem with mixed decision variables, including integer decision variables ( $I$ and $N$ in Eq. (1)), continuous decision variables $\left(E_{E G}^{t}\right.$ in Eq. (1)), and binary decision variables $\left(s_{O N}^{i, t}, s_{O F F}^{i, t}\right.$ in Eq. (1)). In this case, a differential evolution (DE) algorithm is adopted to solve the optimization problem of the upper MG management in this paper.

Compared with the first layer, the optimization problem of the second layer can be considered a simple resources allocation problem. The solution can be directly adopted by the greedy algorithm or random search method. However, from the point of view of the problem, it is necessary to minimize the cost of each EV. Therefore, for reducing the computational time, an efficient greedy strategy combined with stochastic search method is proposed to obtain the near-optimal solution in this paper.

In addition, optimizing energy management at the upper level and energy distribution at the lower level are closely coupled. On the one hand, the optimization of MG resource management performance depends on the battery capacity of EVs. On the other hand, for a given $\mathrm{MG}$, the performance of the underlying layer is affected unless the resulting energy arrangement is optimal.

In summary, in the proposed method, MG optimization management is implemented in the first layer based on the available EV SOC at first. Then, according to the given deployment, it is easy to determine the energy deployment value of the total EVs. By optimizing the energy distribution of the lower layer, the initial value of the next period can be updated. Thus, the lower layer guarantees the feasibility of the upper layer, and the upper layer affects the scheduling performance of the lower layer. Therefore, two-layer joint optimization is realized.

\section{A. DE with the stochastic selection and greedy strategy}

$\mathrm{DE}$ is a population based stochastic optimization technique, which was introduced in 1995 [18], [19]. Because of the advantages, such as easy to use and implement, reliable and fast, DE is a useful optimization technology able to tackle non-differentiable, non-linear and multimodal functions 
promptly. Like other population-based algorithms, the randomly generated individuals are evolved to obtain the optimal solution through operational strategies such as recombination, evaluation and selection. At present, based on the classical DE algorithm, various mutation and crossover strategies, the parameter adaptive scheme has been proposed and successfully used to solve complex problems with different characteristics [17]. Tiwari and Srivastava [20] used the DE algorithm to analyze the energy scheduling problem of MG combined with renewable energy. The operation procedure of the classical DE is shown as follows:

Initialization: As shown in Eq. (11), where $N$ denotes the population size, $g$ defines the generation counter, and $D$ is the dimension of the decision vector.

$$
\begin{aligned}
& P_{x, g}=X_{i, g}, i=0,1, \ldots, N, g=0,1, \ldots, g_{\max } \\
& X_{x, g}=X_{j, i, g}, j=0,1, \ldots, D .
\end{aligned}
$$

During initialization, a set of candidate solutions is created by assigning random values to each decision vector. To guarantee the availability of candidate solutions, the upper and lower bounds of each parameter must be defined before initialization. The initialization of the population is done as follows:

$$
X_{j, i, 0}=\operatorname{rand}_{j, i}[0,1]\left(X_{j, \max }-X_{j, \min }\right)+X_{j, \min }
$$

In Eq. (12), the D-dimensional initialization vectors $X_{j, \max }$ and $X_{j, \min }$ indicate the lower and upper bounds of the parameter vectors $X_{j, i}$. The $\operatorname{rand}_{j, i}[0,1]$ returns a uniformly distributed random number between 0 and 1 . And the subscript $j$ indicates that a new random value is generated for each parameter.

Mutation: A mutation vector $v_{i, g}$ can be generated base on vector $y_{i, g}$ by using a difference vector based mutation, as follows:

$$
v_{i, g}=y_{i, g}+F \cdot\left(x_{r 1, g}-x_{r 2, g}\right),
$$

where indices $r 1$ and $r 2$ are mutually exclusive integers randomly chosen from the population. $F$ is a positive real number that controls the rate at which the population evolves. After mutation, if an element violates the search boundary, it is set to the corresponding bound.

Crossover: To complement the differential mutation search strategy, DE can also employ uniform crossover. In particular, $\mathrm{DE}$ crosses each vector with a mutant vector:

$$
u_{i, j, g}= \begin{cases}v_{i, j, g} & \text { if } \operatorname{rand}_{j}[0,1] \leq C r \text { or } j=j_{\text {rand }} \\ x_{j, i, g} & \text { others }\end{cases}
$$

It can be seen in Eq. (14), the most commonly employed binomial crossover operates on each variable based on crossover rate $\mathrm{Cr}$ and a randomly generated number between 0 and 1 , where $j_{\text {rand }}$ is any randomly chosen natural number in $\{1,2, \cdots, d\}$ with $d$ being the dimension of real-valued decision vectors.

Selection: After mutation and crossover, the objective function values of all trial vectors are evaluated. Then, a selection

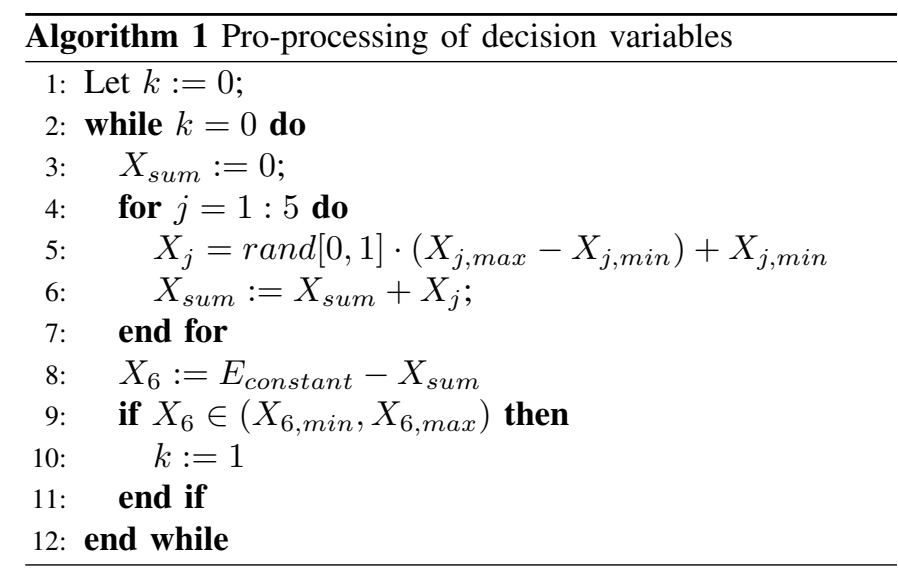

operation is performed. The selection operation can be expressed as follows:

$$
X_{i, G+1}= \begin{cases}U_{i, G} & \text { if } f\left(U_{i, G}\right) \leq f\left(X_{i, G}\right) \\ X_{i, G} & \text { otherwise }\end{cases}
$$

The objective function value of each new vector is compared with the current objective function value. If the objective function value of the new individual is better than the old value, it will be retained in the next generation. Otherwise, the old individual will be retained.

In addition, considering that the high-dimensional problem will affect the initialization speed of the algorithm, this paper pre-processes the decision variables through an equality constraint and random strategy. As shown in Algorithm 1, in this paper, the decision variables $X_{1}$ to $X_{6}$ are comprised of $E_{E G}^{t}$ (the exchange power with the main grid), $E_{E V a g g}^{t}$ (the auxiliary service power from the EV aggregate), $E_{C F G}^{t}$ (the power from CFG) and $E_{E S S}^{n, t}$ (the exchange power of ESS). According to the energy conservation constraint in Eq. (2), the sum of the decision variables is equal to a constant $E_{\text {constant }}$. During initialization, $X_{6}$ is directly and randomly set as any value in its value range, so as to reduce the dimension of the problem and improve the initialization speed.

Also, the greedy strategy and random method are adopted in the second layer to improve the efficiency of energy allocation. The greedy strategy is that the total energy is allocated in order, distributing each car to their current energy ceiling, until the total energy is empty. The random method assigns a random energy to each vehicle in order within their safe range, until the total power is running out.

In this paper, the scheduling strategy is determined according to the ratio of the total dispatch energy and the available energy space of the schedulable vehicle. If the result is greater than $70 \%$, a greedy strategy is used; otherwise, if the result is less than or equal to $70 \%$, a random strategy is used. The main steps of the allocation strategy are shown in Algorithm 2.

In summary, the main procedures of the optimization method are as shown in Algorithm 3. 


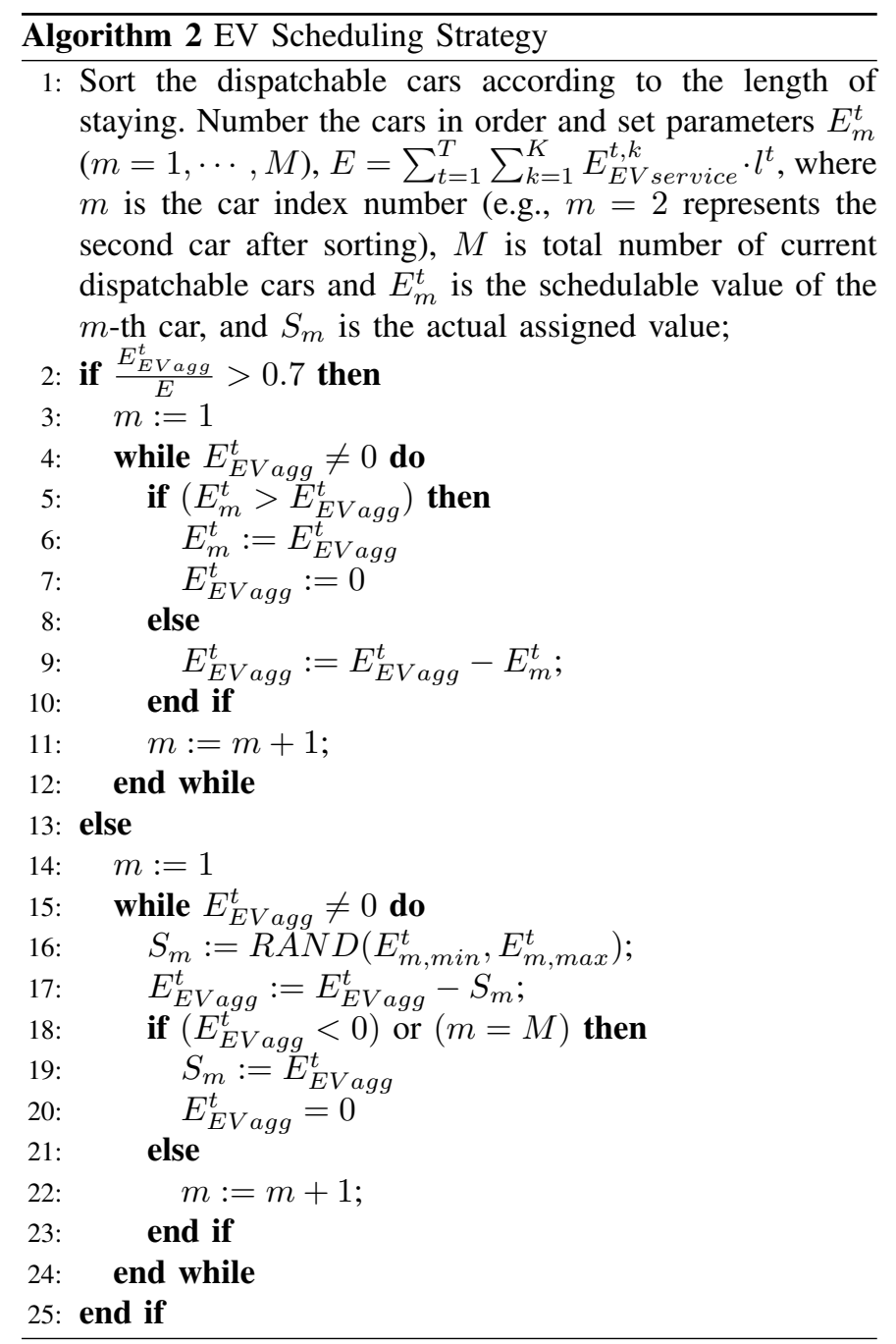

\section{Simulation AND RESUlts}

In this section, a grid-connected RES MG is simulated based on Matlab to provide the experimental environment for evaluating the performance of the proposed management method. The main components and parameters of the simulation model are given in Table II. The operation progress with this case is considered with one hour per time interval along 24 hours. The randomness of MG is not the main research direction of this paper. Therefore, the one day environmental data (including light intensity, wind speed and temperature) as well as the load of MG were randomly selected from local historical data as a case study. The MG load data is shown in Fig. 2.

In addition, to describe EV information mathematically, it is assumed that the arrival time $t_{a r r}^{k}$, departure time $t_{d e p}^{k}$, and EV initial $S O C$ at the arrive time $S O C_{a r r}^{k}$ follow a normal distribution [21]. Therefore, the travel behaviours of $50 \mathrm{EVs}$ are simulated by Monte Carlo simulation. The parameters and setting of EVs are summarized in Table III. The charging demand of each EV is set at $90 \%$ SOC while they are leaving, regardless of the amount of power the vehicle arrives within the simulation. The electric car's access time to the MG was

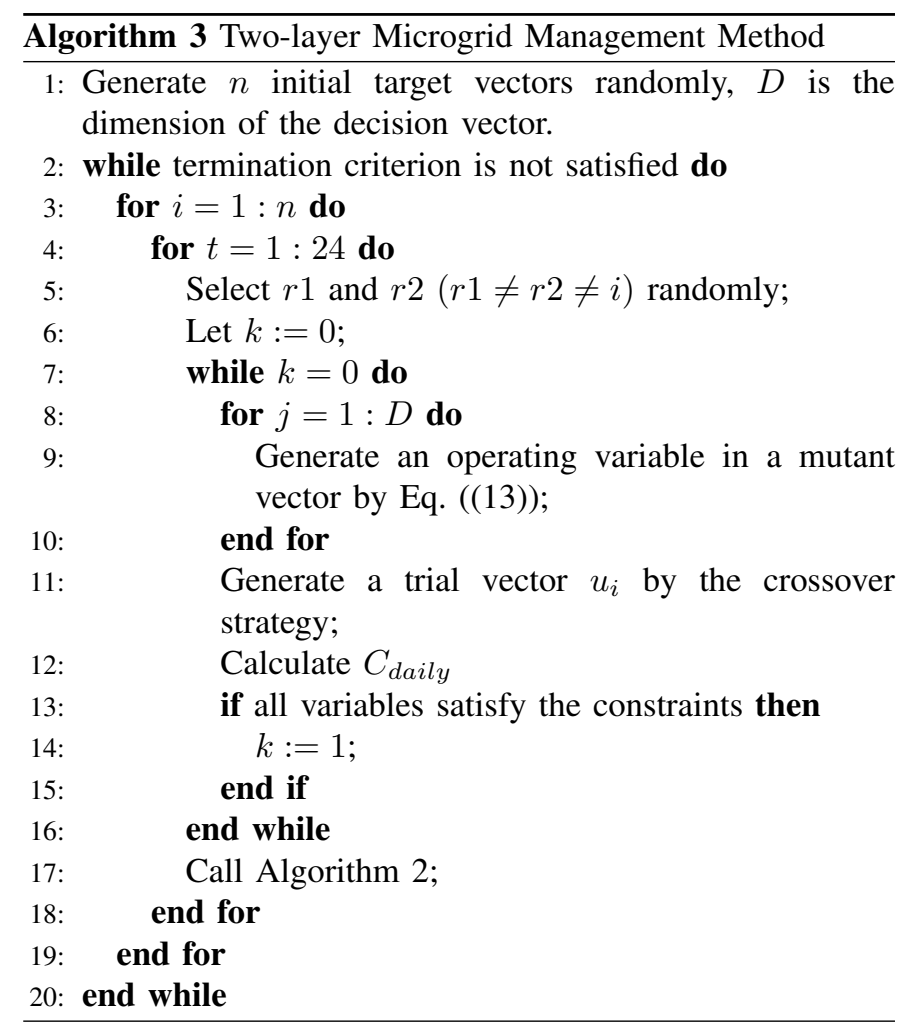

simulated to be the same as the time in the office area, which is between 8 a.m. and 6 p.m. In this article, after the arrival of an EV, the charging time of the EV will be determined immediately according to the predicted electricity price, and the EV will not participate in the auxiliary services of the MG during the charging time.

TABLE II

THE MAIN MG DEVICES IN SIMULATION

\begin{tabular}{c|c|c}
\hline MG components & Capacity(KW) & Units \\
\hline Micro gas turbine & 65 & 1 \\
\hline Wind turbine & 30 & 1 \\
\hline Photovoltaic generation & 0.2 & 300 \\
\hline ESS & 400 & 1 \\
\hline
\end{tabular}

TABLE III THE PARAMETERS OF EVS

\begin{tabular}{c|c|c}
\hline \multirow{4}{*}{ EV } & Main parameter & Values \\
\cline { 2 - 3 } & Battery capacity & $30 \mathrm{kwh}$ \\
\cline { 2 - 3 } & Simulation number & 50 \\
\cline { 2 - 3 } & SOC lower/upper limits & $10 / 90$ \\
\cline { 2 - 3 } & Preset require SOC & 90 \\
\hline
\end{tabular}

As mentioned in the previous section, a DE algorithm is adopted as the solution method for the upper layer complex optimization problem. The parameters of the DE algorithm, $F$ and $C r$, are set to 0.9 . The number of iterations is set to 200 . The initial population size is 20 . Considering the solving characteristics of different algorithms, a PSO algorithm was added to the simulation experiment for comparison. The parameters $c 1$ and $c 2$ of PSO were set to 0.45 , and the population size 


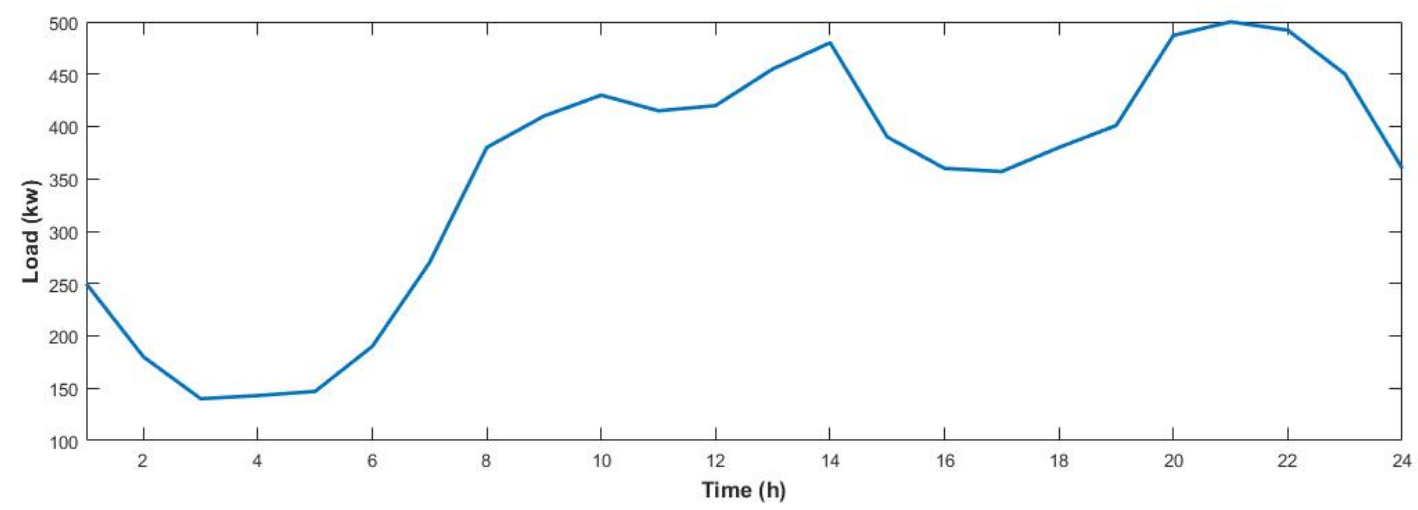

Fig. 2. Predicted 24-hour microgrid load.

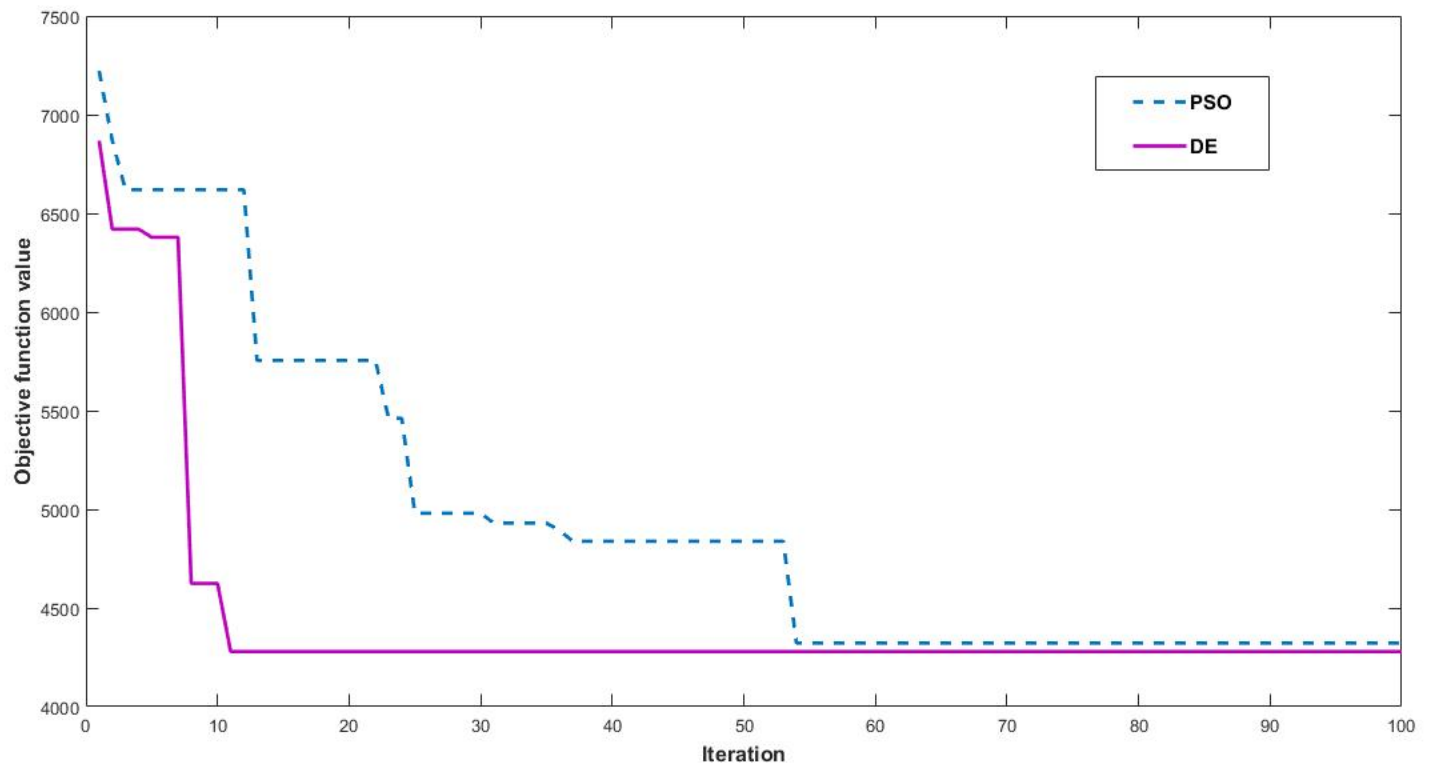

Fig. 3. Comparison between DE and PSO.

and the iteration number were set to the same values as those for the DE algorithm.

TABLE IV

EV OWNER COST UNDER MGS WITH/WITHOUT AUXILIARY SERVICE

\begin{tabular}{c|c|c}
\hline \multirow{2}{*}{ EV ID } & \multicolumn{2}{|c}{ THE COST \$ } \\
\cline { 2 - 3 } & with auxiliary service & without auxiliary service \$ \\
\hline EV 01 & 1.4257 & 1.9023 \\
\hline EV 25 & 1.0024 & 1.1321 \\
\hline EV 50 & 1.4376 & 1.4523 \\
\hline
\end{tabular}

In this research, the economic benefits of $\mathrm{MG}$ operation depend on the optimization results of the upper layer of the proposed model. Therefore, the performance of the optimization algorithm can determine the quality of the scheme. Through the comparison between PSO and DE in Fig. 3, both algorithms can find the optimal solution in a short time and realize the improvement of $\mathrm{MG}$ economic benefits. However, DE is slightly superior to PSO in terms of the accuracy and speed of solution.

Although the total amount of energy allocated in the lower layer comes from the result of the optimal scheduling in the upper layer. This part is only a relatively simple resource allocation problem, but different methods bring different effects. This section compares the effects of different allocation strategies in different scenarios. Considering the limited space of this article, only three cars were selected for the comparison results of the experiment, as shown in Table IV. It can be seen from the table that the proposed strategy can effectively adapt to the energy distribution needs of various scenarios. Revenue from ancillary services when the energy ratio is equal to $96 \%$.

In addition, in the simulation, we also noticed the performance of the allocation strategy and its impact on the fairness 
of the car owner. When the proportion of distributable energy is large, the owner's cost is significantly reduced. However, when the energy ratio is less than $70 \%$, there is no guarantee that all owners will benefit. Also if we only use random search, in the case of energy ratio greater than $90 \%$, the whole resource allocation needs to occupy a lot of time. Therefore, an appropriate resource allocation strategy is closely related to the interests of car owners.

\section{CONCLUSiON}

This paper proposes a two-layer optimization management method for a RES MG with EVs scheduling. The first-layer optimizes the energy management of MG from the perspective of MG economy by combining the energy storage characteristics of EVs; the second layer, the reasonable allocation of energy can reduce the charging cost of each $\mathrm{EV}$, from the view of EVs owners. The proposed two-layer management method is formulated and solved by a DE algorithm with greedy and stochastic strategies. The main outcomes are as follows:

- A MG management model is established to try to guarantee the economic interests of different interest subjects. The model explains that EVs and MG restrict and benefit from each other, and can quickly adapt to different scenarios by modifying the corresponding parameters. Also, simulation results show that both $\mathrm{MG}$ and $\mathrm{EV}$ users can benefit from this method, which also potentially promotes the $\mathrm{V} 2 \mathrm{G}$ participation of EV owners. It has good portability and practical application value.

- In addition, an evolutionary algorithm, DE, is used as a solution to the problem to participate in the evaluation of the model. In the solving process, the energy distribution strategy of EVs is also analyzed and discussed.

In the future work, from the perspective of the application of $\mathrm{MG}$, the influence of the random characteristics of MG on the model should be fully considered. In addition, the coordination and scheduling effect of this model in the future intelligent comprehensive energy system should also be studied. From the view of the solution method, through further analysis of the different scenarios, the improvement of solution strategy and algorithm can be investigated by the aid of the proposed model.

\section{REFERENCES}

[1] J. Keirstead, M. Jennings, and A. Sivakumar, "A review of urban energy system models: Approaches, challenges and opportunities," Renewable and Sustainable Energy Reviews, vol. 16, no. 6, pp. 3847-3866, aug 2012.

[2] A. Tavakoli, M. Negnevitsky, D. T. Nguyen, and K. M. Muttaqi, "Energy Exchange Between Electric Vehicle Load and Wind Generating Utilities," IEEE Transactions on Power Systems, vol. 31, no. 2, pp. 12481258, mar 2016.

[3] L. Jian, Y. Zheng, X. Xiao, and C. Chan, "Optimal scheduling for vehicle-to-grid operation with stochastic connection of plug-in electric vehicles to smart grid," Applied Energy, vol. 146, pp. 150-161, may 2015.

[4] H. Morais, T. Sousa, Z. Vale, and P. Faria, "Evaluation of the electric vehicle impact in the power demand curve in a smart grid environment," Energy Conversion and Management, vol. 82, pp. 268-282, jun 2014.
[5] A. Rabiee, M. Sadeghi, J. Aghaeic, and A. Heidari, "Optimal operation of microgrids through simultaneous scheduling of electrical vehicles and responsive loads considering wind and PV units uncertainties," Renewable and Sustainable Energy Reviews, vol. 57, pp. 721-739, may 2016.

[6] Y. Zhao, M. Noori, and O. Tatari, "Vehicle to Grid regulation services of electric delivery trucks: Economic and environmental benefit analysis," Applied Energy, vol. 170, pp. 161-175, may 2016.

[7] S. Deilami, A. S. Masoum, P. S. Moses, and M. A. S. Masoum, "Real-Time Coordination of Plug-In Electric Vehicle Charging in Smart Grids to Minimize Power Losses and Improve Voltage Profile," IEEE Transactions on Smart Grid, vol. 2, no. 3, pp. 456-467, sep 2011.

[8] S. Aghajani and M. Kalantar, "A cooperative game theoretic analysis of electric vehicles parking lot in smart grid," Energy, vol. 137, pp. 129-139, oct 2017.

[9] K. M. Tan, V. K. Ramachandaramurthy, and J. Y. Yong, "Integration of electric vehicles in smart grid: A review on vehicle to grid technologies and optimization techniques," Renewable and Sustainable Energy Reviews, vol. 53, pp. 720-732, jan 2016.

[10] D. Wu, D. C. Aliprantis, and L. Ying, "Load Scheduling and Dispatch for Aggregators of Plug-In Electric Vehicles," IEEE Transactions on Smart Grid, vol. 3, no. 1, pp. 368-376, mar 2012.

[11] J. R. Pillai and B. Bak-Jensen, "Integration of Vehicle-to-Grid in the Western Danish Power System," IEEE Transactions on Sustainable Energy, jan 2010

[12] E. Mortaz and J. Valenzuela, "Optimizing the size of a V2G parking deck in a microgrid," International Journal of Electrical Power \& Energy Systems, vol. 97, pp. 28-39, apr 2018.

[13] Y. Cao, S. Tang, C. Li, P. Zhang, Y. Tan, Z. Zhang, and J. Li, "An Optimized EV Charging Model Considering TOU Price and SOC Curve," IEEE Transactions on Smart Grid, vol. 3, no. 1, pp. 388-393, mar 2012. [Online]. Available: http://ieeexplore.ieee.org/document/5978239/

[14] H. Kamankesh, V. G. Agelidis, and A. Kavousi-Fard, "Optimal scheduling of renewable micro-grids considering plug-in hybrid electric vehicle charging demand," Energy, vol. 100, pp. 285-297, apr 2016.

[15] A. Kavousi-Fard, A. Abunasri, A. Zare, and R. Hoseinzadeh, "Impact of plug-in hybrid electric vehicles charging demand on the optimal energy management of renewable micro-grids," Energy, vol. 78, pp. 904-915, dec 2014.

[16] D. Wang, X. Guan, J. Wu, P. Li, P. Zan, and H. Xu, "Integrated Energy Exchange Scheduling for Multimicrogrid System With Electric Vehicles," IEEE Transactions on Smart Grid, vol. 7, no. 4, pp. 17621774, jul 2016.

[17] L. Lakshminarasimman and S. Subramanian, "Applications of Differential Evolution in Power System Optimization," in Advances in Differential Evolution. Berlin, Heidelberg: Springer Berlin Heidelberg, 2008, pp. 257-273.

[18] K. V. Price, R. M. Storn, and J. A. Lampinen, Differential evolution : a practical approach to global optimization. Springer, 2005.

[19] U. K. Chakraborty, Advances in differential evolution. Springer Verlag, 2008.

[20] N. Tiwari and L. Srivastava, "Generation scheduling and micro-grid energy management using differential evolution algorithm," in 2016 International Conference on Circuit, Power and Computing Technologies (ICCPCT). IEEE, mar 2016, pp. 1-7.

[21] Z. Liu, Y. Chen, R. Zhuo, and H. Jia, "Energy storage capacity optimization for autonomy microgrid considering chp and ev scheduling," Applied Energy, vol. 210, pp. 1113-1125, 2018 